\title{
C2 vertebral corpectomy for kyphosis malunion of remote dens fracture with atlantoaxial ankylosis and myelopathy - case report at twelve years follow-up
}

\section{Drew Bednar}

McMaster University Faculty of Health Sciences Hamilton, Ontario CANADA

\begin{abstract}
Background Context: Late myelopathy from dens fracture non-union is rare and management in this case was greatly challenged by complete arthritic ankyloses $\mathrm{C1} / 2$

Purpose: We report a case of ankylosed dens malunion in kyphosis developing progressive myelopathy decades after injury and requiring unique salvage surgery.

Study Design/Setting: An academic spine care center. Patient Sample - the patient was a 75-yearold female at presentation.

Outcome Measures: Clinical myelopathy, X-ray alignment, CT scan.

Methods - careful C2 corpectomy immediately distal to the odontoid process was successful in reversing myelopathy and preserving stability. This case is presented without funding support and the patient has given her permission for her case to be presented.

Results: At twelve years the patient was free of myelopathy and had stable cervical alignment.

Conclusions: Apex decompression for myelopathy from cervical kyphosis can be achieved and successful long-term in rare cases where realignment osteotomy may not be feasible.
\end{abstract}

A 75-year old woman had undergone posterior wireinstrumented instrumented atlantoaxial fusion for displaced odontoid base fracture at the age of 32 years. She presented with an 8-months history of progressive numbness in all four limbs with nondisabling loss of fine motor control and dexterity in the hands. There was occasional instability of gait on stairs but not yet requiring walking aids and without falls. There was stable mild chronic neck pain and no incident trauma.

Physical exam revealed a normally ambulatory senior with brisk hyperreflexia in all four limbs and positive Hoffman's and Babinski's signs, but no clonus.

X-ray (Figure 1) suggested a stable and intact posterior wire/ graft fusion from $\mathrm{C} 1$ to $\mathrm{C} 2$ in kyphosis. Flexion/extension views confirmed stability.

CT scan (Figures 2A, 2B) confirmed a continuous posterior fusion mass at $\mathrm{C} 1 / 2$ with established kyphotic malunion of previous dens base fracture. There were also complete ankyloses of the lateral atlantoaxial joints, the atlantodental joint and the antlanto-occipital joint with incomplete extraarticular ankylosis at $\mathrm{C} 2 / 3$.

MRI (Figure 3) showed kyphotic tenting/thinning of the spinal cord at the postero-superior body of $\mathrm{C} 2$. There was neither posterior compression nor any anterior compression behind the dens itself.

\section{Case Management}

Transoral odontoidectomy was not an option because spinal cord compression was not caused by the odontoid but by the vertebral body of $\mathrm{C} 2$ below it. Also, maxillary excursion was limited by arthritic temporomandibular joints and judged insufficient for transoral exposure.

Realignment osteotomy at $\mathrm{C} 1 / 2$ was considered and declined for potential risk to the vertebral arteries.

Subtraction osteotomy (excision) of the prominent posterior aspect of the $\mathrm{C} 2$ vertebral body was performed via a high SmithPeterson approach as recently described by Yang et al [1]. The patient was positioned supine in light (5 pounds) GardnerWells traction with an inflatable pillow [2] placed immediately posterior to the neck to maximize cervical lordosis. Traction tape was applied under the mentum to give slight incremental extension proximally and lift the mandible away from the surgical field. Incision was at $\mathrm{C} 4$ to allow for the required obliquity of instrumentation access. Deep to platysma blunt dissection was used to approach C2 directly and so create a working channell into which a standard self-retaining cervical retractor (Trimline(C) Medtronic Canada, Mississauga) could be placed for protection of the surrounding structures. The infrajacent $\mathrm{C} 2 / 3$ disc space and the malunited fracture site at the dens base defined respectively the caudal and cephalad limits of resection. The uncovertebral articulations $\mathrm{C} 2 / 3$ were preserved to protect the vertebral arteries and to provide anatomic continuity $\mathrm{C} 2 / 3$ in the anterior column.

At surgery in 2003 neither image guidance nor spinal cord monitoring were available at our hospital. The posteroinferior dens base was collinear to the posterosuperior cortex of the $\mathrm{C} 2$ pars providing a radiographic guide to the site of compression and required decompression. 
Citation: Bednar D. C2 vertebral corpectomy for kyphosis malunion of remote dens fracture with atlantoaxial ankylosis and myelopathy case report at twelve years follow-up. J Neurol Neurorehabil Res. 2016;1(2):3-6.

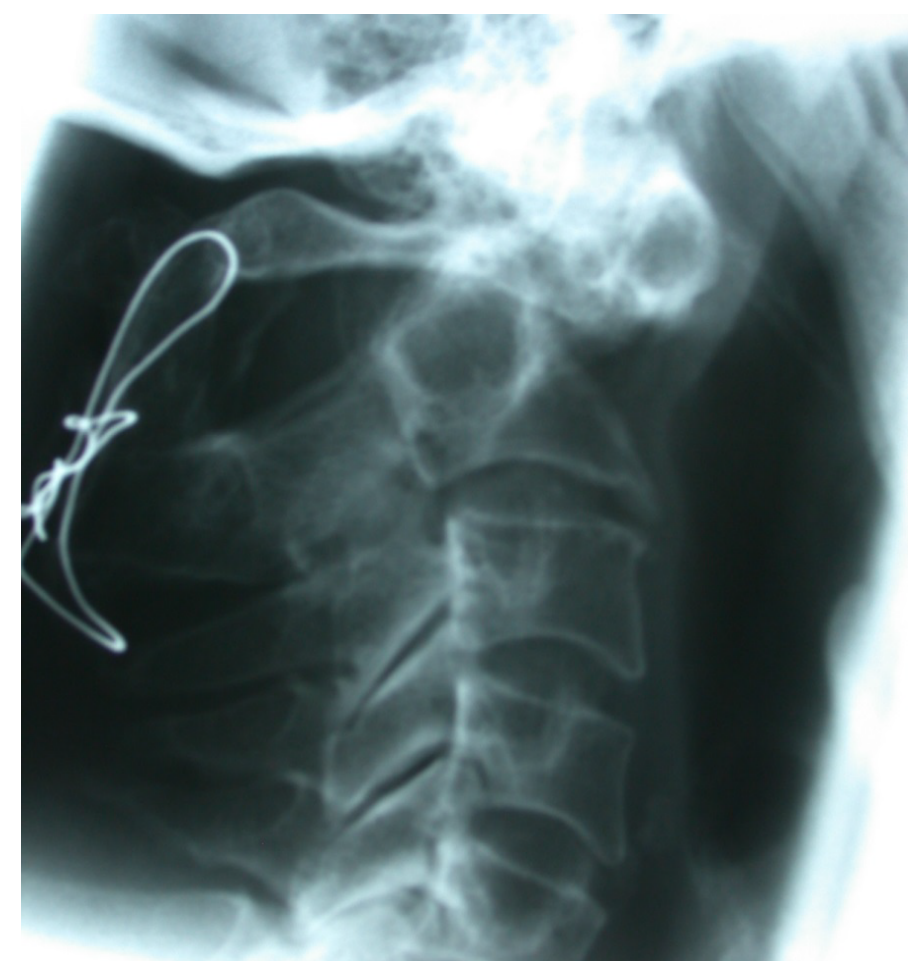

Figure 1: Lateral X-ray at presentation showing old posterior fusion $\mathrm{C} 1 / 2$ in kyphosis

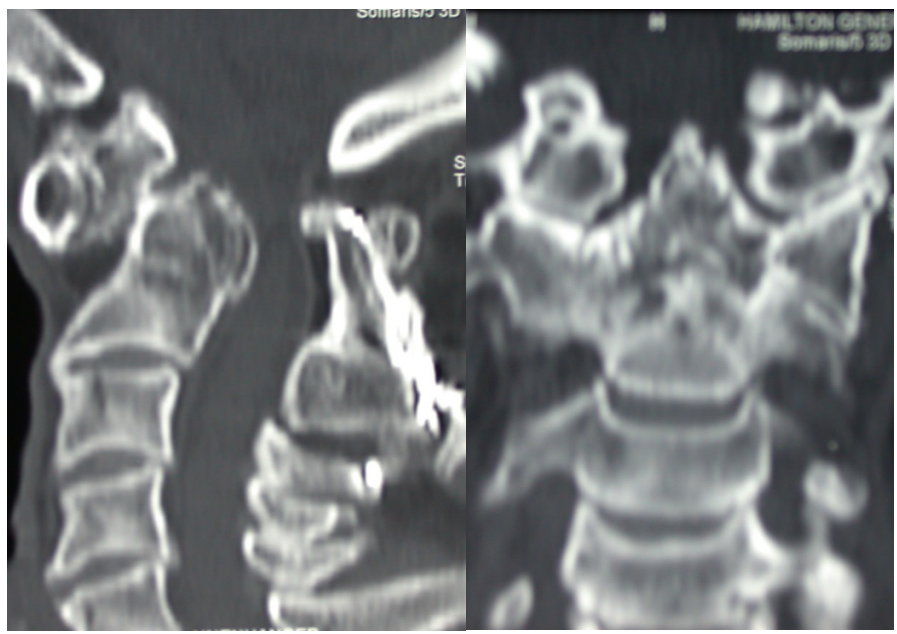

MRI shows cord narrowing over the apex of kyphosis with no posterior compression.

Figure 2: AP (right) and Lateral (left) CT images confirm solid posterior fusion and complete ankyloses of the lateral occipitoatlantal and atlantoaxial joints.

Resection was performed with a high-speed drill (Figure 4) until the posterior cortex was penetrated. Remaining middle column bone was then reflected forward into the osteotomy defect with fine curettes (Figure 5) and a dural hook (Figure 6) until the dura could be visualized across the full extent of the decompression site.

Postoperatively there was immediate and complete relief of myelopathy. The patient wore a hard cervical collar when ambulatory for a three-month period. At six months X-rays confirmed no change in cervical alignment and the asymptomatic patient was discharged from active care.

Seven years later she was involved in a low-energy motor vehicle accident and evaluated in the emergency room with $\mathrm{X}$-rays and a CT scan confirming stable cervical alignment and adequate decompression at the osteotomy site.
At fourteen years she remains free of myelopathy.

\section{Discussion}

In this case excessive fixed cervical kyphosis caused cord compression through acute angulation and decompression could be achieved either by realignment of kyphosis or anterior resection of the apex of the bony kyphosis at the vertebral body C2. Neurological symptoms were of recent onset and quickly progressive so observation was not an option. Interestingly (and in retrospect) both the $\mathrm{O} / \mathrm{C} 2$ angle of Ota et al [3] at $15^{\circ}$ and the occipitocervical angle of Riel et al (4) at $118^{\circ}$ were within normal limits in this case.

It is unclear why our patient developed myelopathy several decades after her fracture repair though there are precedents [5, 6. No remote imaging or reports were available to define the immediate postoperative alignment after fracture repair but the 


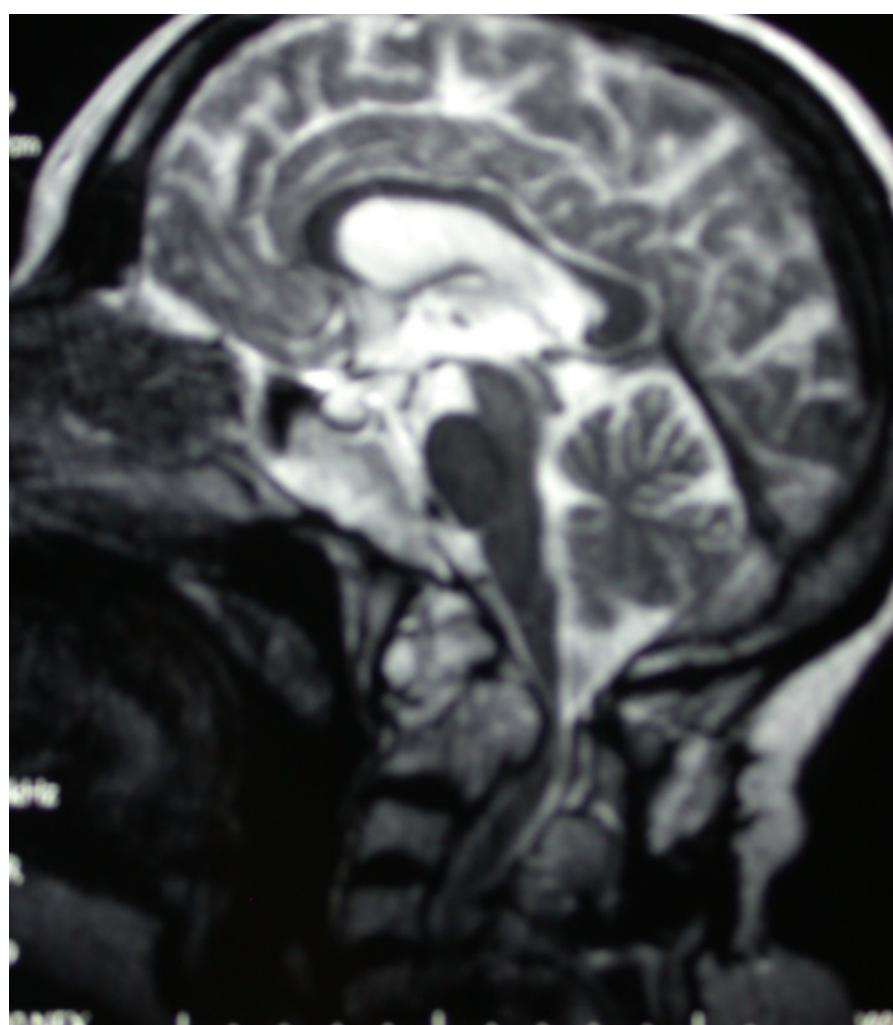

Figure 3: MRI shows cord narrowing over the apex of kyphosis with no posterior compression.

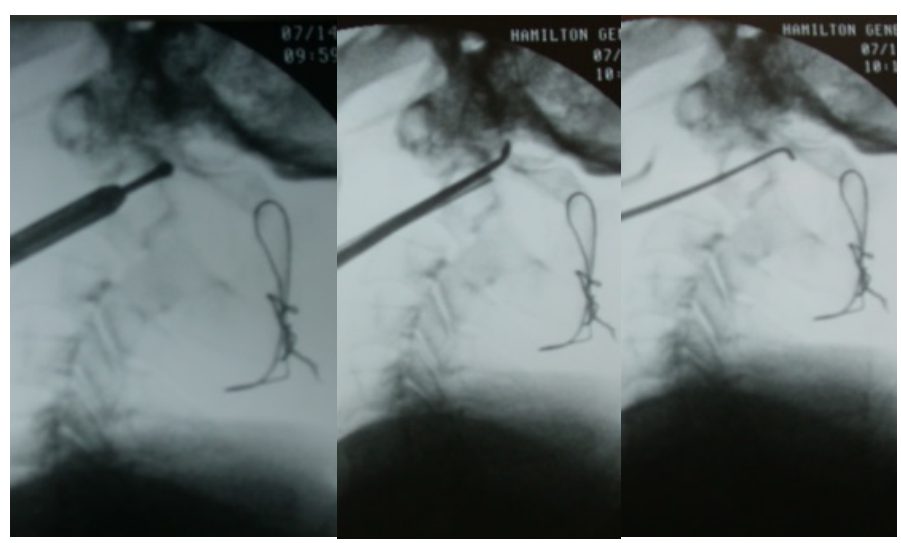

Figure 4: Operative fluoroscopy; corpectomy (left), curettage (middle) and probing of the successfully decompressed canal (right).

position of the patients' $\mathrm{C} 1 / 2$ interspinous wire (spanning $\mathrm{C} 1$ to $\mathrm{C} 2$ in good juxtaposition) suggests that the baseline fracture alignment had not changed significantly.

Realignment osteotomy would have required resection through the vertebral artery foramena bilaterally and was not considered safely possible. It remains unprecedented.

\section{Conclusion}

Apex decompression for myelopathy from cervical kyphosis can be achieved and successful

long-term in rare cases where realignment osteotomy may not be feasible. Careful resection of the $\mathrm{C} 2$ vertebral body's posterior cortical prominence with preservation of the uncovertebral joints proved safe and long-term effective in this case.

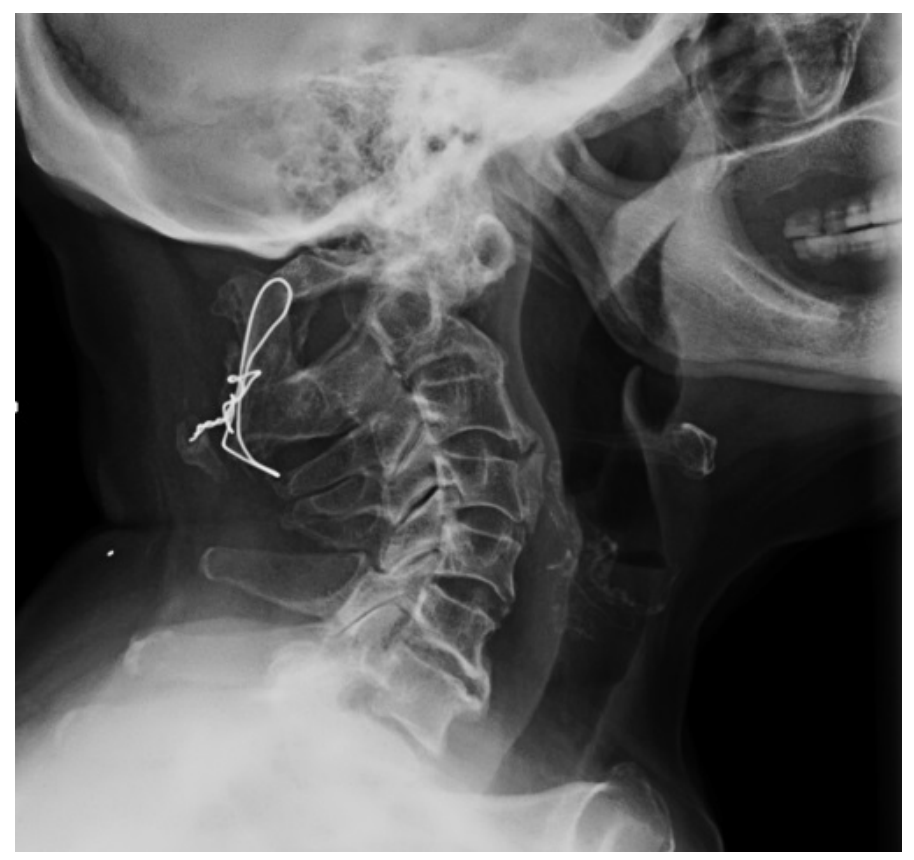

Figure 5: X-ray after MVC at seven years after surgery confirms stable alignment.

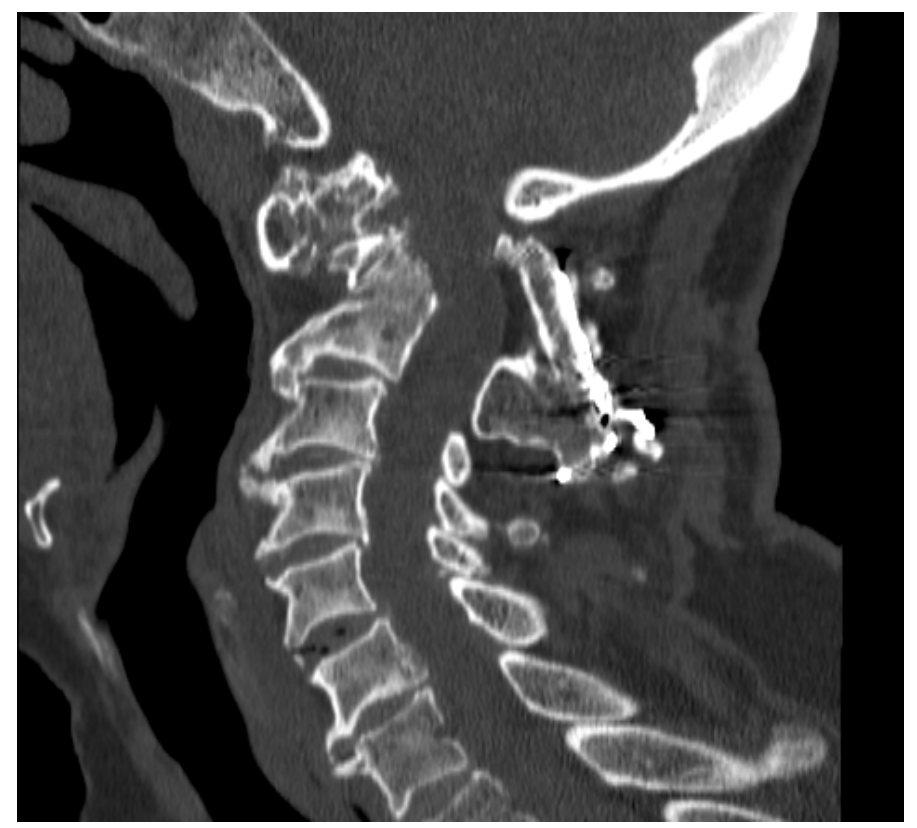

Figure 6: CT scan after MVC at seven years after surgery confirms significant decompression.

\section{References}

1. Yang JS, Chu L, Xiao FT, et al. Anterior retropharyngeal approach to $\mathrm{C} 1$ for percutaneous vertebroplasty under C-arm fluoroscopy. The Spine Journal 2015;15:539-45.

2. Alsunbul WA, Algird AR, Sanjer MF, et al. Inflatable Device for Intraoperative Control Cervical Extension in Cervical Surgery. Can J Neurol Sci 2014;41:293-95.

3. Ota M, Neo M, Aoyama T, et al. Impact of the O-C2 Angle on the Oropharyngeal Space in Normal Patients. Spine 2011;36(11):E720-6.

4. Riel RU, Lenke LG, Riew KD. Measurement of a Posterior 
Citation: Bednar D. C2 vertebral corpectomy for kyphosis malunion of remote dens fracture with atlantoaxial ankylosis and myelopathy case report at twelve years follow-up. J Neurol Neurorehabil Res. 2016;1(2):3-6.

Occipitocervical Fusion Angle. J Spinal Disord Tech 2010;23(1):27-9.

5. Rudzki JR, Lenke LG, Blanke K, et al. Pseudoarthrosis of a thirty-nine-year-old dens fracture causing myelopathy - A case report. J Bone Joint Surg 2004;86:2509-13.
6. Fairholm D, Lee ST, Lui TN (1996)Fractured odontoid: the management of delayed neurological symptoms. Neurosurgery. 1977;38:38-43.

\section{*Correspondence to:}

Keisuke Kawata

Indiana University-Bloomington, Department of Kinesiology

Bloomington

E-mail: kkawata@indiana.edu

Tel: 8128566035 